\title{
Measurement of the Intra-ventricular Mechanical Dyssynchrony in Cardiac Magnetic Resonance Images
}

\author{
Zhenzhou Wang \\ College of electrical and electronic engineering, Shandong University of Technology, Zibo City, Shandong Province, and \\ China \\ Email: wangzz@sdut.edu.cn
}

How to cite this paper: Zhenzhou Wang (2020). Measurement of the Intra-ventricular Mechanical Dyssynchrony in Cardiac Magnetic Resonance Images. Journal of Artificial Intelligence and Systems, 2, 98-117.

https://doi.org/10.33969/AIS.2020.21007.

Received: December 24, 2019

Accepted: February 13, 2020

Published: February 20, 2020

Copyright $(2020$ by author(s) and Institute of Electronics and Computer. This work is licensed under the Creative Commons Attribution International License (CC BY 4.0).

http://creativecommons.org/licenses/by/4.0/

\begin{abstract}
Measurement of the mechanical dyssynchrony of the ventricle is important for the cardiac resynchronization therapy (CRT) and the evaluation of patients with heart failures. So far, feature-tracking cardiovascular magnetic resonance (FT-CMR) has become the most popular method for measuring the mechanical dyssynchrony. FT-CMR calculates the strain values based on the pre-defined segments of the ventricle and thus it might miss some important regional information. In this paper, we propose a new approach to measure the intra-ventricular mechanical dyssynchrony based on the uniformly sampled points from all the regions of the ventricle. Accordingly, the proposed approach will not miss any regional information of the ventricle. In addition, the proposed approach is fully automatic and not depedent on the training sets. It calculates the mechnical dyssynchrony rapidly and determines whether the tested case is normal or patient immdiately. The proposed approach was validated with 40 tested cases (20 normal cases and 20 patient cases). Experimental results showed that the fully automatic intra-ventricular mechanical dyssynchrony measurement approach achieved $100 \%$ diagnosis accuracy for the left ventricle (LV) and achieved 95\% diagnoisis accuracy for the right ventricle (RV).
\end{abstract}

\section{Keywords}

Dyssynchrony, measurement, Image processing, Cross correlation, Strain, Feature-tracking cardiovascular magnetic resonance.

\section{Introduction}

Cardiovascular magnetic resonance (CMR) has an established and continuously expanding role in tissue characterization and is the modality of choice for accurate evaluation of biventricular morphology and function. CMR provides diagnostic information that is often expressed numerically. It measures cardiac muscle motion and deformation by post-processing of standard gray-scale B-mode or cine imaging, resulting in easier access and wider availability. However, it has limited sensitivity for identifying regional myocardial impairment. On the other hand, some wall motion evaluations were done on a subjective basis that suffers a substantial error rate. In the past decades, several CMR techniques have been developed to measure 
cardiac muscle motion and deformation and FT-CMR was the most popular and promising one [1-5]. FT-CMR provides quantitative information about myocardial deformation by calculating the myocardial strain (MS) that measures the degree of deformation of a myocardial segment from its initial length to its maximum length. Longitudinal strain calculates the longitudinal shortening from the base to the apex. Radial strain calculates the myocardial deformation towards the center of the LV's cavity. Circumferential strain calculates LV myocardial fiber shortening along the circumference in the short axis view.

At present time, researchers are working toward applying FT-CMR in clinical scenarios. Some research work was conducted to determine the normal range of the LV strain [6-8] and some research work was conducted to assess the RV strain and dyssynchrony [9-11]. From the conducted research work, it is seen that MR strain values calculated by different techniques or vendors are significantly different. Hence, it is required to develop reference standard from each technique or vendor for clinical use. The inconsistency is mainly caused by the use of different post-processing techniques from different vendors. However, it also reflects the instability of the way to calculate the strain values to some extent. The strain values are calculated based on the pre-defined segments of the ventricle and thus it might miss some important regional information. Most importantly, it is almost impossible to align the pre-defined segments consistently and accurately in different frames and slices due to the torsion and displacement of the heart. Consequently, the calculated strain values might be very inaccurate for some tested cases. In this paper, we propose an approach to calculate the mechanical dyssynchrony between the motions of the sampled points on extracted boundaries of the ventricles and the volumetric cycle of the ventricle. Compared to the limited number of the segments used for strain calculation, 100 evenly sampled points in each slice are used to calculate the mechanical dyssynchrony, which could obtain all the regional information of muscle motion and deformation adequately.

Besides the FT-CMR method, many other methods had been proposed for measuring the mechanical dyssynchrony in CRT in the 1990s [12-15]. However, none of them could measure the mechanical dyssynchorony occurred in the ventricle accurately as reported in [13-15]. In recent years, the demand for measuring the mechanical dyssynchrony has been increasing significantly. It has been widely recognized that the mechanical dyssynchorony occurred in the ventricle has become a major predictor for the CRT. To address this critical problem, we have proposed a new approach to measure the mechanical dyssynchorony occurred in the left ventricle [16]. However, the proposed approach could not measure the mechanical dyssynchorony occurred in the right ventricle robustly. In this paper, we aim to propose a more robust approach to measure the mechanical dyssynchorony occurred in both the left ventricle and the right venticle accurately.

\section{The Proposed Approach}

During each systolic and diastolic cardiac cycle, the heart contracts and expands periodically. For a normal heart, all the cardiac muscles contract and expand synchronously with the cardiac cycle. When some cardiac muscles do not contract and expand synchronously with the cardiac cycle, the mechanical dyssynchrony occurs. The proposed approach samples hundreds of points uniformly on the cardiac muschles to measure the mechanical dyssynchrony while FT-CMR divide the whole cardiac muschles into 13 regions and measure the mechanical 
dyssynchrony of these regions. Thus the proposed approach could measure the mechanical dyssynchrony more thoroughly and accurately than FT-CMR.

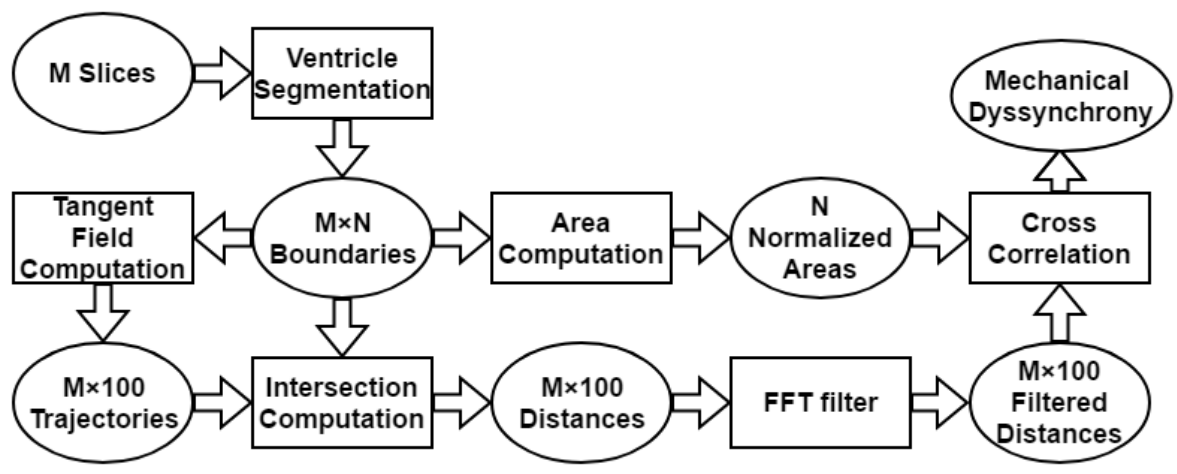

Fig.1. Flowchart of the proposed approach.

Fig. 1 shows the proposed approach's flowchart. M slices of the tested cases are selected to calculate the mechanical dyssynchrony between the motion of $\mathrm{M} \times 100$ sampled points and the variation of the ventricular volume. The $\mathrm{M}$ slices are segmented by the ventricle segmentation method automatically. $\mathrm{M} \times \mathrm{N}$ boundaries of the ventricle from the $\mathrm{M} \times \mathrm{N}$ frames are obtained. From the obtained boundaries, the center of the ventricle and the area of the ventricle are computed. Based on the number of the frames contained in each slice, $\mathrm{N}$ areas of the ventricle in different frames are calculated and normalized. The center of the ventricle is dilated to form an inner boundary. The largest obtained boundary by ventricle segmentation is dilated to form the outer boundary. The tangent field between the outer boundary and the inner boundary is calculated. In each slice, 100 correspondence trajectories originate from the ventricle's center and stop at the sampled points on the outer ventricular boundary along the direction of the tangent filed. The intersections between the 100 correspondence trajectories and the $\mathrm{N}$ obtained boundaries by segmentation are calculated for each slice and $\mathrm{M} \times 100$ distances from the intersections to the center of the ventricle are obtained. A Fast Fourier Transformation (FFT) based low pass filter is used to remove the outliers that might be caused by flaws of the CMR imaging technique, the acquired image or image processing algorithms. The $\mathrm{M} \times 100$ distances from the intersections to the center of the ventricle in the $\mathrm{N}$ frames of the tested case form a cycle. The normalized areas in the $\mathrm{N}$ frames of the tested case also form a cycle. The lag time between these two cycles are calculated by cross correlation and defined as mechanical dyssynchrony. When there is the intra-ventricular mechanical dyssynchrony occurring at one sampled point, a lag relative to the reference time occurs. Accordingly, the mechanical dyssynchrony is computed as the lag. The calculated lag is normalized in the range of $[0,1]$. A threshold is determined on-line or off-line to distinguish the patient cases from the normal controls. The threshold is selected as 0.3 because even for the normal controls, it is rare for all sampled points of the ventricular wall to contract at the same rate strictly.

\subsection{Ventricle Segmentation}

The flowchart of the ventricle segmentation method [17-18] is shown in Fig. 2. Firstly, the slope difference distribution (SDD) of the LV ROI or the RV ROI is computed. The peaks and valleys of SDD are determined. Based on the positions 
and magnitudes of the peaks, the means of three pixel classes corresponding to the bright blood pools, the grey myocardium and the dark surrounding structures are computed as described in [17]. The threshold selection process is divided into two situations and is summarized as Algorithm 1. With the optimal threshold, the LV ROI or the RV ROI is segmented. The morphology operations are used to select the blob with the largest area from the segmentation result as the LV segment or the RV segment. The LV segment is transformed to a convex hull while the RV segment is smoothed by morphology operations during the post-processing stage. Then, the boundary of the LV segment or the RV segment is extracted and filtered. The filtered LV boundary or RV boundary is used for mechanical dyssynchrony measurement.

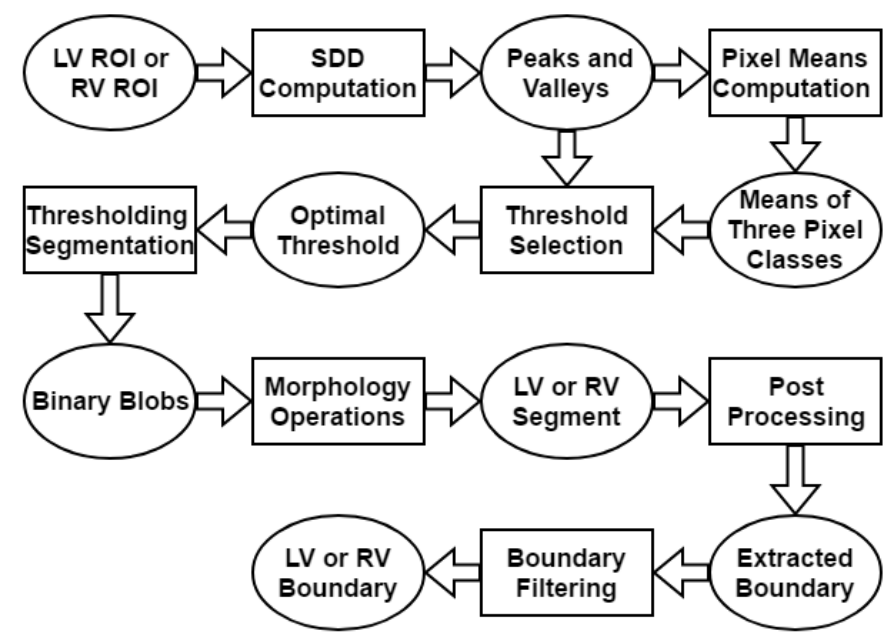

Fig.2. Flowchart of the automatic ventricle segmentation method.

Algorithm 1 SDD threshold selection

Input: The valleys of slope difference distribution $V_{i} ; i=1,2, \ldots, N_{V}$ and the peaks of slope difference distribution $P_{j} ; j=1,2, \ldots, N_{P}$.

Output: The selected threshold $T$

Threshold selection:

1), Compute the center of the dark pixel class $u_{1}$, compute center of the grey pixel class $u_{2}$ and compute the center of the bright pixel class $u_{3}$.

2), Compute the gray-scale values that the valleys of SDD correspond to by the following Equation:

$G_{x}{ }^{V}=\left\{x \mid S(x)=V_{x}\right\}, x=1,2, \ldots, N_{V}$

Situation 1: $u_{1}$ is smaller than or equal to 22

3), Compute the number of the gray-scale values of $G_{x}{ }^{V}$ between $u_{2}$ and $u_{3}$ by the following equation:

$X=\left\{x \mid u_{2}<G_{x}{ }^{V}<u_{3}\right\}, x=1,2, \ldots, N_{V}$

4), If the size of $X$ is null, the threshold is computed as:

$T=\left(u_{2}+u_{3}\right) / 2$

5), If the size of $X$ is equal or smaller than 2, the threshold is computed as: $T=\min \left(\left[G_{\min (x)}{ }^{V}, G_{\max (x)}{ }^{V}\right]\right)$

6), If the size of $X$ is greater than 2, the threshold is computed as:

$T=G_{x T}{ }^{V}, x T=\left\{x \mid V_{x}=\max \left(V_{x}\right)\right\}$

Situation 2: $u_{1}$ is greater than 22 
7), Compute the number of the gray-scale values of $G_{x}{ }^{V}$ between $u_{1}$ and $u_{2}$ by the following equation:

$X=\left\{x \mid u_{1}<G_{x}{ }^{V}<u_{2}\right\}, x=1,2, \ldots, N_{V}$

8), The threshold is computed as:

$T=G_{x T}{ }^{V}, x T=\left\{x \mid V_{x}=\max \left(V_{x}\right)\right\}$

Result: the selected threshold $T$.

Fig. 3 demonstrates the LV segmentation process based on the automatically localized LV ROI and Fig. 4 demonstrates the RV segmentation process based on the automatically localized RV ROI. As can be seen, both the LV and the RV are segmented robustly.

In recent years, deep learning has achieved great success in object classification and semantic image segmentation [19-32]. However, deep learning could only generate a prediction map of the target instead of the accurate boundary of the target. To measure the mechanical dyssynchrony robustly, the boundaries of the ventricles must be segmented accurately. Consequently, the deep learning based image segmentation methods are not suitable for segmenting the LV and the RV in this study. In addition, the SDD based ventricle segmentation method does not require any training sets and thus it is more efficient for clinical usage.

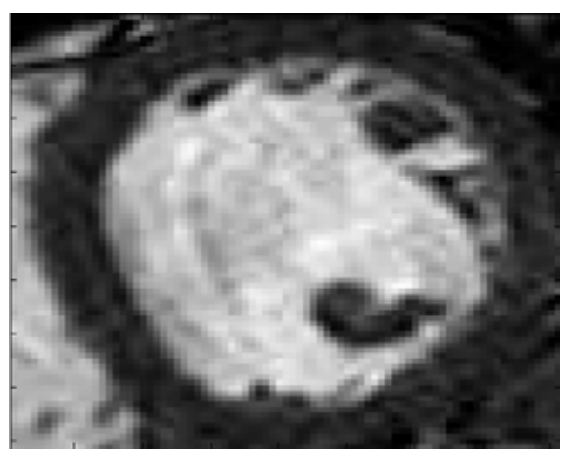

(a)

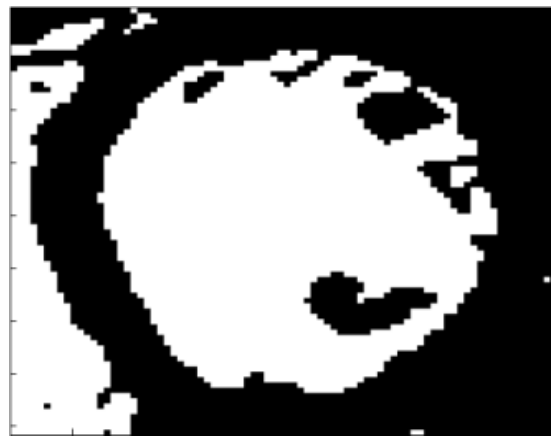

(c)

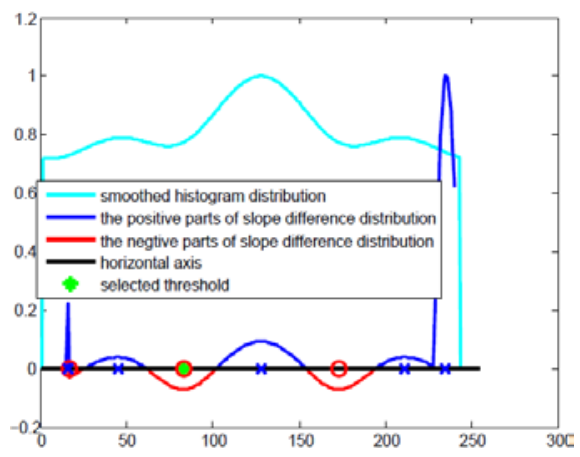

(b)

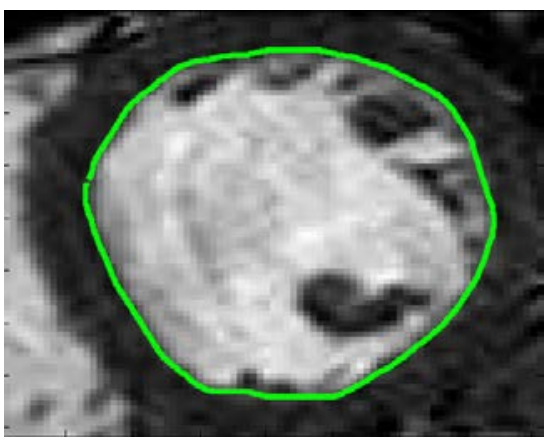

(d)

Fig.3. Demonstration of segmenting the LV in the LV ROI. (a) The LV ROI; (b) The threshold selection process for the LV ROI; (c) The segmentation result of LV ROI; (d) The identified LV boundary. 


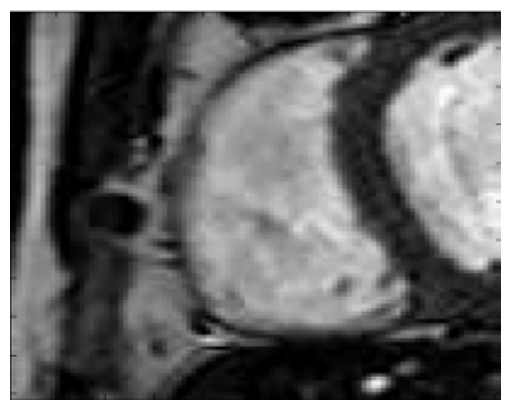

(a)

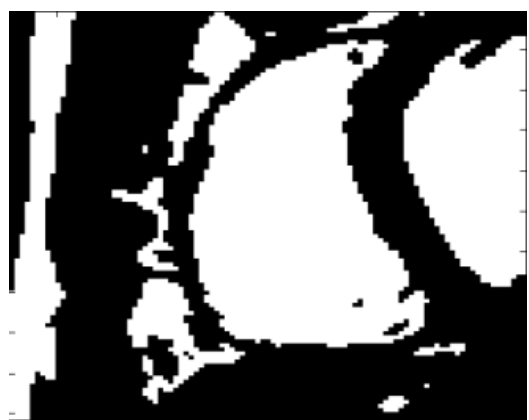

(c)

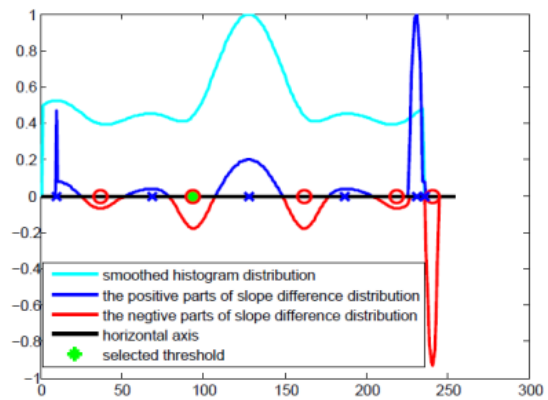

(b)

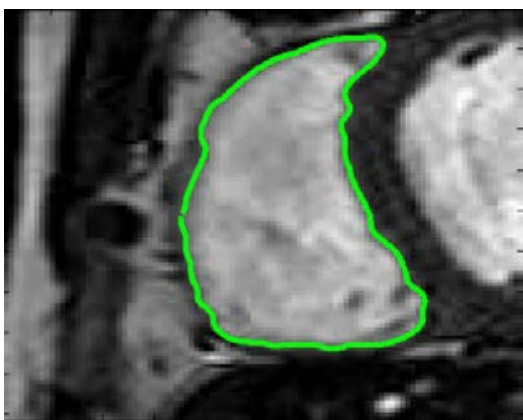

(d)

Fig.4. Demonstration of segmenting the RV in the RV ROI. (a) The RV ROI; (b) The threshold selection process for the RV ROI; (c) The segmentation result of RV ROI; (d) The identified RV boundary.

\subsection{Mechanical Dyssnychrony Computation}

The flowchart of the intra-ventricular mechanical dyssynchrony measurement method is shown in Fig. 5. $M$ middle slices in a tested case are used to generate $M \times N$ boundaries by the automatic SDD ventricle segmentation method. From the $M \times N$ boundaries, $N$ normalized areas are computed. Then tangent field in the largest ventricle boundary of each selected slice is computed and $M \times 100$ boundaries (trajectories) are generated. The intersections between $M \times 100$ trajectories and the $M \times N$ ventricle boundaries are computed to obtain $M \times N \times 100$ intersection points. In each slice, the distances between these $N \times 100$ intersection points and the centroid of the ventricle in this slice are computed. Accordingly, a total of $M \times N \times 100$ distances are obtained for all the $M$ slices. These $M \times N \times 100$ distances are filtered by a FFT filter with the bandwidth 2 and then their cross correlations with the previously computed $N$ normalized areas are computed. The weighted dyssynchrony level (WDL) is generated by cross correlation and it is compared with a predefined threshold to determine if the tested case is a normal control or a patient. 


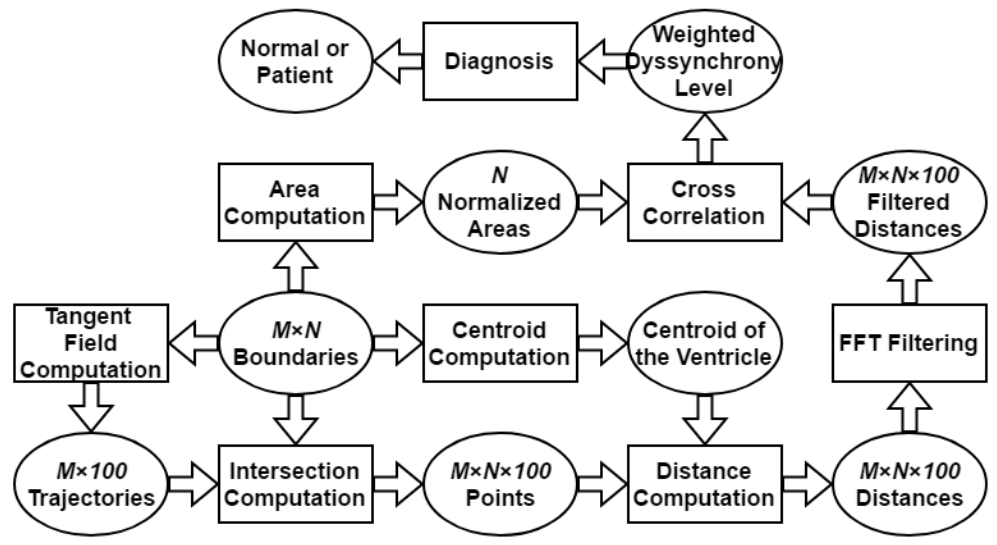

Fig.5. Flowchart of the automatic intra-ventricular mechanical dyssynchrony measurement method.

Fig. 6 demonstrates the process of measuring the intra-ventricular mechanical dyssynchrony. As can be seen, the WDL of the LV is significantly lower than the WDL of the RV in the same tested case.

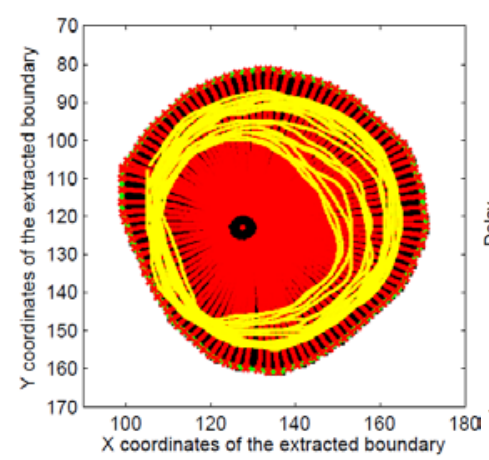

(a)

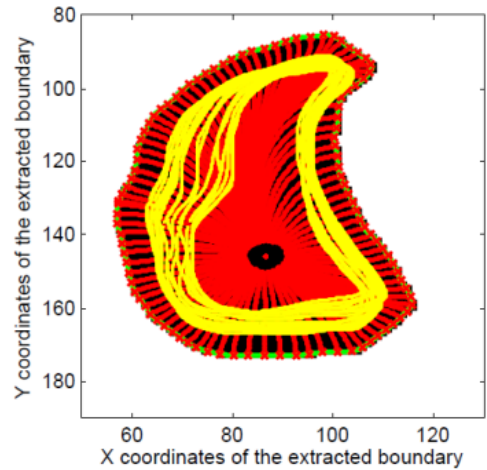

(c)

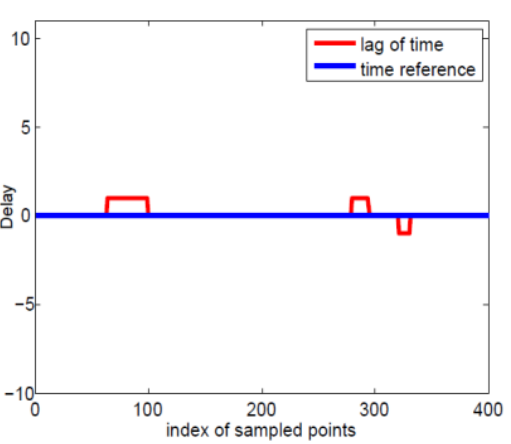

(b)

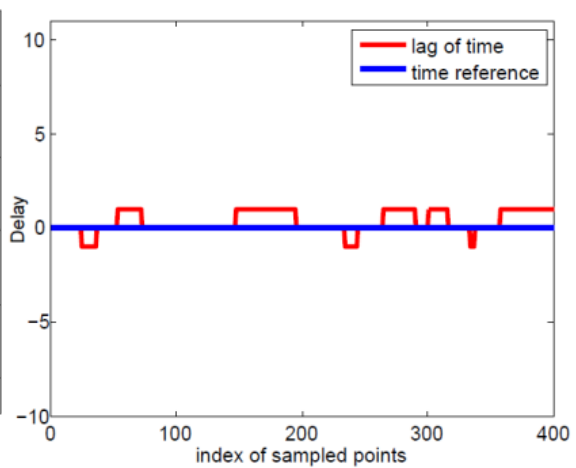

(d)

Fig.6. Demonstration of measuring the mechanical dyssynchrony. (a) The extracted 20 boundaries of the LV denoted in yellow lines and the 100 trajectories denoted in red lines; (b) The weighted dyssynchrony level computed with four adjacent center slices of LV; (c) The extracted 20 boundaries of the RV denoted in yellow lines and the 100 trajectories denoted in red lines; (d) The weighted dyssynchrony level computed with four adjacent center slices of RV. 


\subsection{The Proposed Registration Based Mechanical Dyssynchrony Measurement Method}

As stated in the conclusion section of [13], the torsion and displacement of the ventricle might decrease the mechanical dyssynchrony measurement accuracy and a registration method might be able to solve the problem. Hence, we propose a registration method to align the ventricles in different frames and calculate the mechanical dyssynchrony based on the boundaries of the aligned ventricles. The flowchart of the proposed registration method is shown in Fig. 7. The centroids $\left(x_{c}^{i}, y_{c}^{i}\right), i=1,2, \ldots, N$ of all the boundaries are computed by Eq. (6). The angle $\theta_{i}, i=1,2, \ldots, N$ of each boundary is computed as the angle of the long axis of this boundary. The differences between the centroid of the largest boundary and the centroids of other boundaries are computed as:

$$
\left(\Delta x_{i}, \Delta y_{i}\right)=\left(x_{c}^{i}-x_{c}^{L}, y_{c}^{i}-y_{c}^{L}\right), i=1,2, \ldots, N
$$

The differences between the angles of the largest boundary and the angle of other boundaries are computed as:

$$
\Delta \theta_{i}=\theta_{i}-\theta_{L}, i=1,2, \ldots, N
$$

The points $\left(x_{j}^{i}, y_{j}^{i}\right), j=1,2, \ldots, J$ on the $i$ th boundary are transformed by the following registration matrix.

$$
\left[\begin{array}{c}
X_{j}^{i} \\
Y_{j}^{i}
\end{array}\right]=\left[\begin{array}{cc}
\cos \Delta \theta_{i} & \sin \Delta \theta_{i} \\
-\sin \Delta \theta_{i} & \cos \Delta \theta_{i}
\end{array}\right]\left[\begin{array}{l}
x_{j}^{i} \\
y_{j}^{i}
\end{array}\right]+\left[\begin{array}{c}
\Delta x_{i} \\
\Delta y_{i}
\end{array}\right]
$$

where $\left(X_{j}^{i}, Y_{j}^{i}\right), j=1,2, \ldots, J$ dnotes the point on the $i$ th registrated boundary and $J$ is the total number of the points on this boundary.

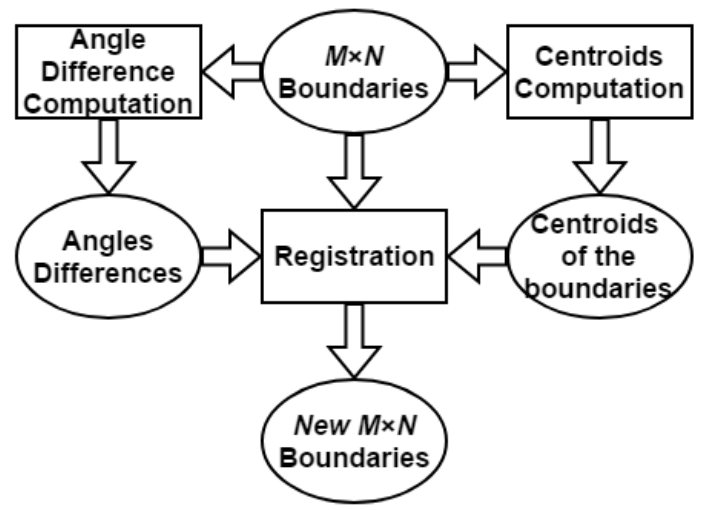

Fig.7. Flowchart of the proposed automatic registration method.

The $M \times N$ boundaries used for dyssynchrony measurement are replaced with the $M \times N$ registered boundaries. The mechanical dyssynchrony is measured again according to the flowchart shown in Fig. 5. Fig. 8 shows the measured mechanical dyssynchrony ater registration for comparison with the measured mechanical dyssynchrony shown in Fig. 6 without registration. As can be seen, the registration reduces the WDL significantly. 


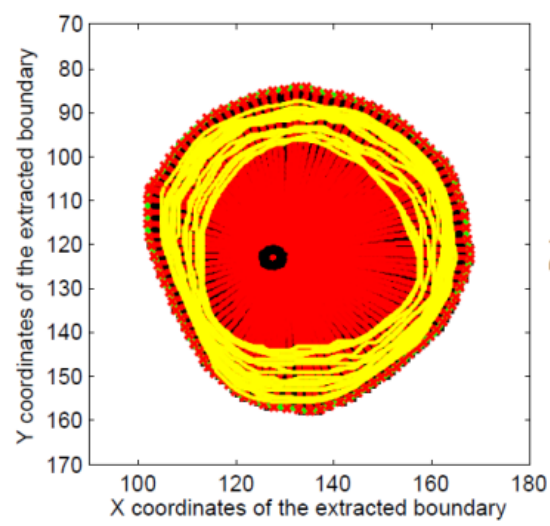

(a)

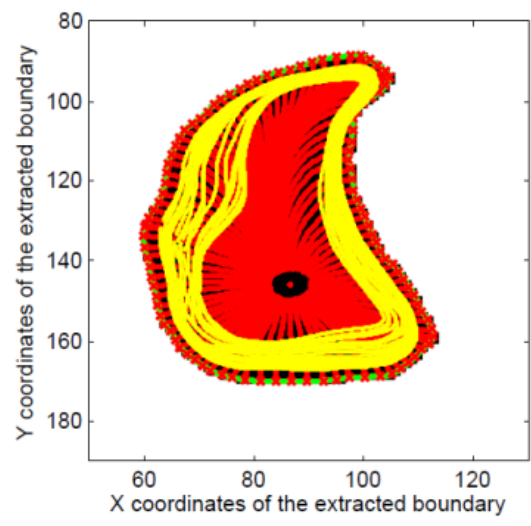

(c)

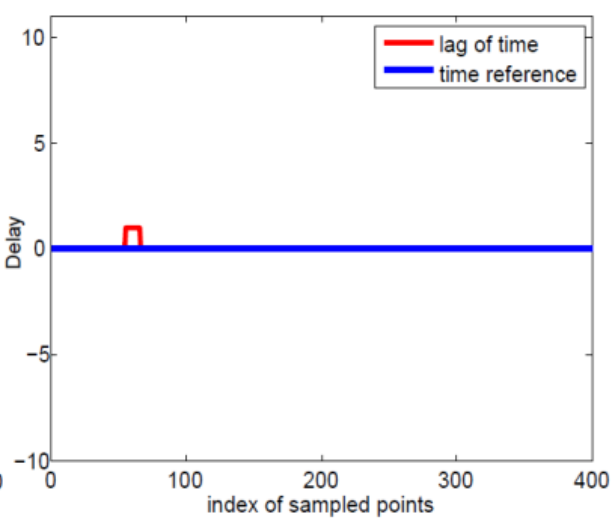

(b)

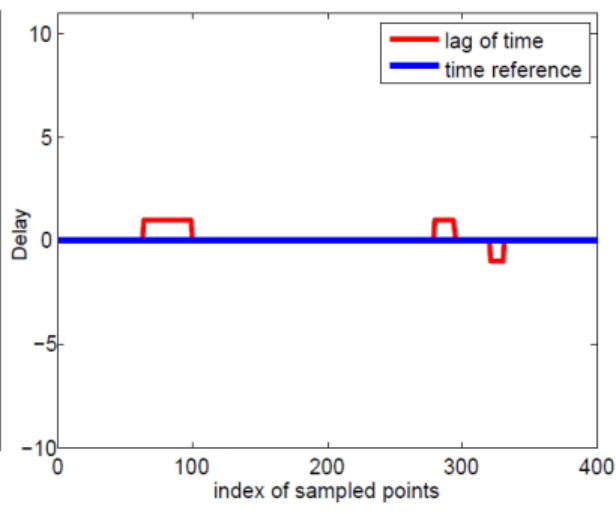

(d)

Fig.8. Demonstration of measuring the mechanical dyssynchrony after ventricle registration. (a) The extracted 20 boundaries of the LV denoted in yellow lines and the 100 trajectories denoted in red lines; (b) The weighted dyssynchrony level computed with four adjacent center slices of LV; (c) The extracted 20 boundaries of the RV denoted in yellow lines and the 100 trajectories denoted in red lines; (d) The weighted dyssynchrony level computed with four adjacent center slices of RV.

\section{Results}

Twenty cases of CMR images were obtained from 20 patients with heart failure and 20 cases of CMR images were obtained from 20 healthy volunteers without history of cardiac disease with a 1.5T Philips Intera scanner. The steady-state free procession (SSFP) short-axis cine images were acquired during 10-15 second breath-holds using a 5-element phased array cardiac coil. Contiguous $8-10 \mathrm{~mm}$ slices were acquired at 20 or 30 frames per cardiac cycle. Acquisition parameters were as follows: acquired matrix size $=192 \times 256$, reconstructed matrix size $=$ $256 \times 256$, field of view $(\mathrm{FOV})=370 \mathrm{~mm}$, flip angle $=65^{\circ}, \mathrm{TR}=4 \mathrm{msec}$ and $\mathrm{TE}$ $=2 \mathrm{msec}$.

For the ventricle segmentation method, there are two parameters, the bandwidth of the DFT low-pass filter $W$ and the fitting number $N$ need to be tuned for optimal segmentation. We use the open accessible dataset from [17] to determine the 
optimal SDD parameters by sensitivity analysis. Both the determined optimal bandwidth $W$ and the determined optimal fitting number $N$ equal 11 .

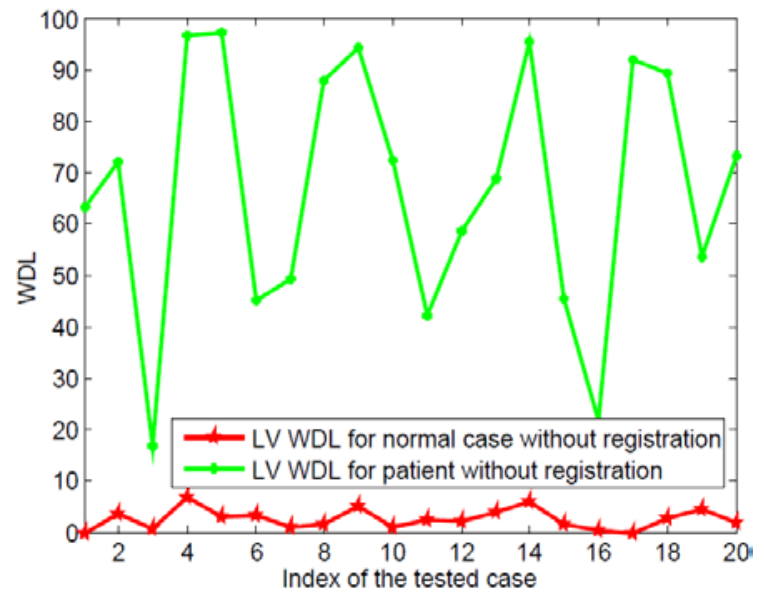

(a)

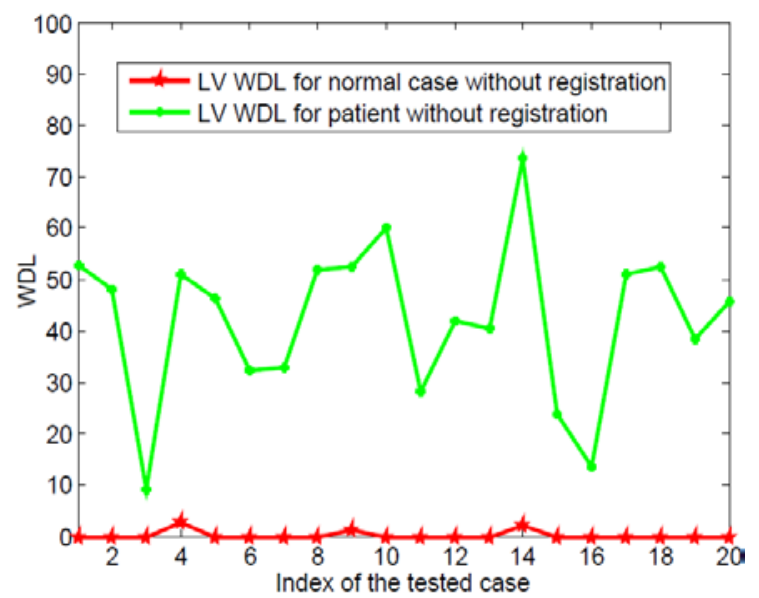

(b)

Fig.9. The measured mechanical dyssynchrony of the LV for 20 normal controls and 20 patients (a) The mechanical dyssynchrony is measured without ventricle registration; (b) The mechanical dyssynchrony is measured with ventricle registration.

We measure the mechanical dyssynchrony of both the LV and the RV for the 20 patients and 20 normal controls. The measured mechanical dyssynchrony is computed as WDL values and they are shown in Fig. 9 for the LV and in Fig. 10 for the RV respectively. In Fig. 9 (a), the WDL values of the LV are computed by the mechanical dyssynchrony measurement method without registration. In Fig. 9 (b), the WDL values of the LV are computed by the mechanical dyssynchrony measurement method with registration. As can be seen, the normal controls and the patients could be differentiated robustly with $100 \%$ accuracy by the computed WDL values no matter the ventricle is registered or not. After ventricle registration, the WDL values of the normal controls are close to zero, which is more accurate. The WDL values of the RV computed by the mechanical dyssynchrony measurement method without registration for the 40 tested cases are shown in Fig. 
10 (a) and the WDL values of the RV computed by the mechanical dyssynchrony measurement method with registration for the 40 tested cases are shown in Fig. 10 (b). As can be seen, the normal controls and the patients could not be differentiated with $100 \%$ accuracy. However, the ventricle registration helps to improve the accuracy of the mechanical dyssynchrony measurement approach from $90 \%$ to $95 \%$. With 100\% LV diagnosis accuracy and 95\% diagnosis accuracy, the proposed approach is able to help CRT and other clinical applications of heart failure evaluations positively.

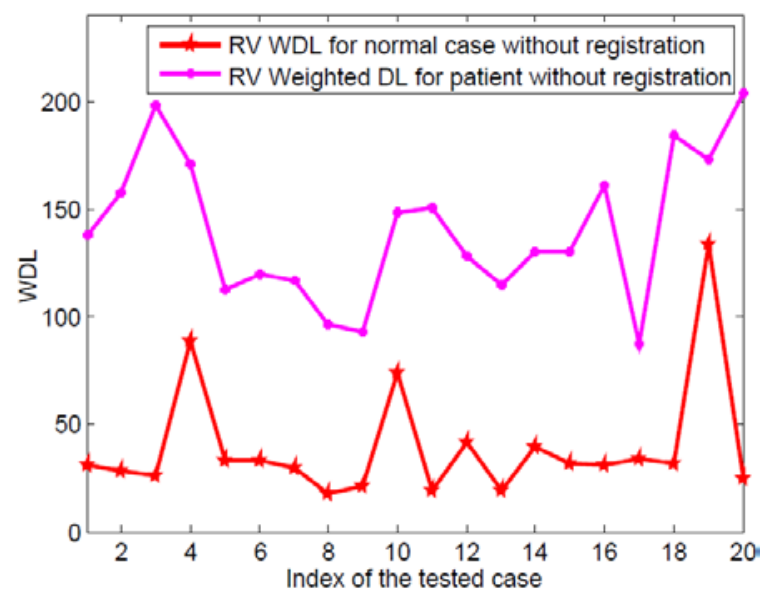

(a)

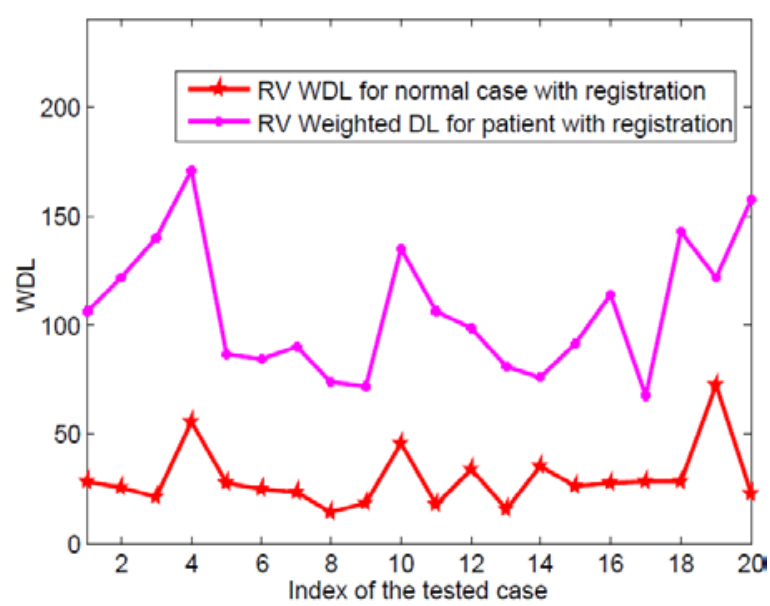

(b)

Fig.10. The measured mechanical dyssynchrony of the RV for 20 normal controls and 20 patients (a) The mechanical dyssynchrony is measured without ventricle registration; (b) The mechanical dyssynchrony is measured with ventricle registration.

Fig. 11 compares the results of computing the mechanical dyssynchrony for the LV of a normal control by the method proposed in [16] and by the approach proposed in this paper. Fig. 11 (a) shows the computed correspondence trajectories and the identified boundaries for one middle slice of the normal control by the method proposed in [16]. Fig. 11 (b) shows the cycles of the filtered distances that are computed from the intersections of the identified boundaries and correspondence 
trajectories by the method proposed in [16]. Fig. 11 (c) shows the cycle of the 20 areas computed from the 20 identified boundaries shown in Fig. 11 (a). Fig. 11 (d) shows the computed mechanical dyssynchrony from four middle slices of the normal control by the method proposed in [16]. Fig. 11 (e) shows the computed correspondence trajectories and the identified boundaries for one middle slice of the normal control by the proposed approach. Fig. 11 (f) shows the cycles of the filtered distances that are computed from the intersections of the identified boundaries and correspondence trajectories by the proposed approach. Fig. 11 (g) shows the cycle of the 20 areas computed from the 20 identified boundaries shown in Fig. 11 (e). Fig. 11 (h) shows the computed mechanical dyssynchrony from four middle slices of the normal control by the proposed approach. Compared to the mechanical dyssynchrony measured by the method proposed in [16], the mechanical dyssynchrony measured by the proposed approach is more accurate.

We compare the results of computing the mechanical dyssynchrony for the LV of a patient case by the method proposed in [16] and by the proposed approach in Fig. 12. It is seen that the proposed approach is more accurate than the method proposed in [16]. We also compare the results of computing the mechanical dyssynchrony for the RV of a normal control and the RV of a patient case by the method in [16] and by the proposed approach in Fig. 13 and Fig. 14 respectively. As can be seen, the proposed approach is more accurate.

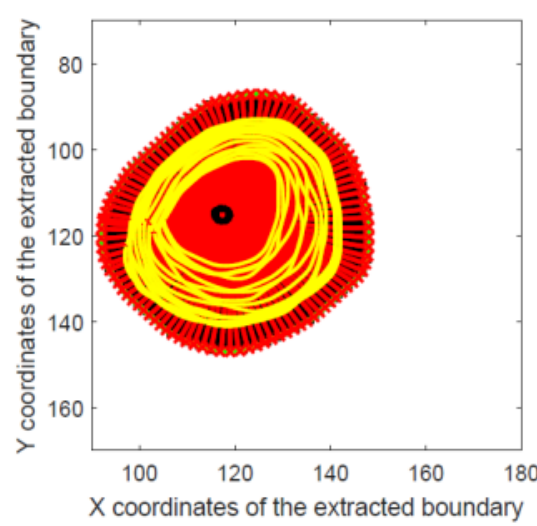

(a)

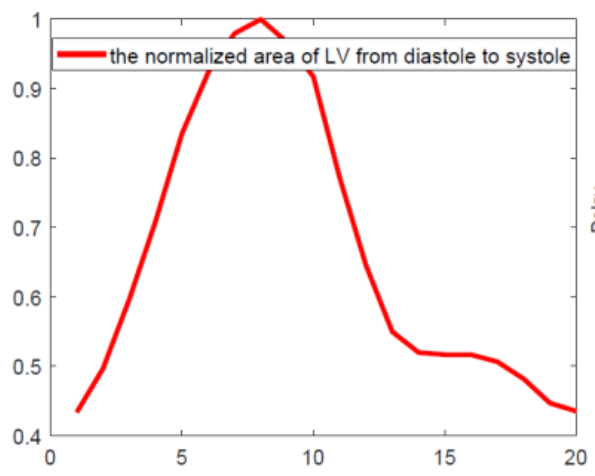

(c)

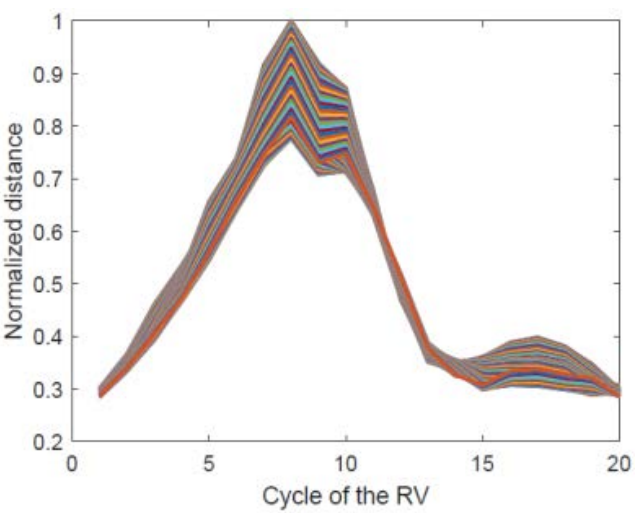

(b)

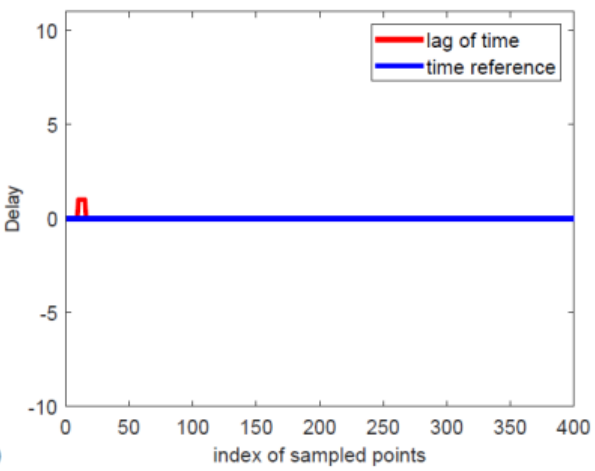

(d) 


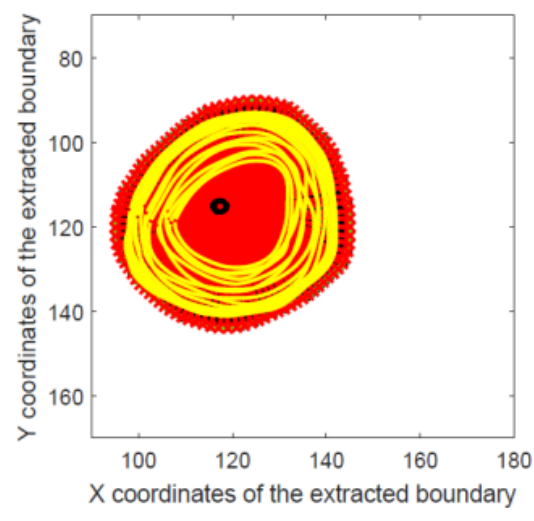

(e)

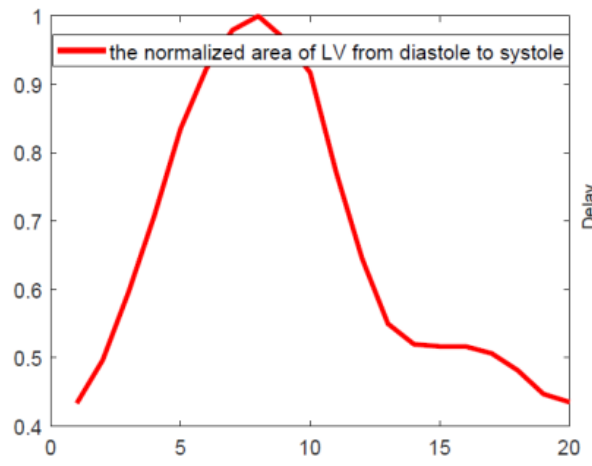

(g)

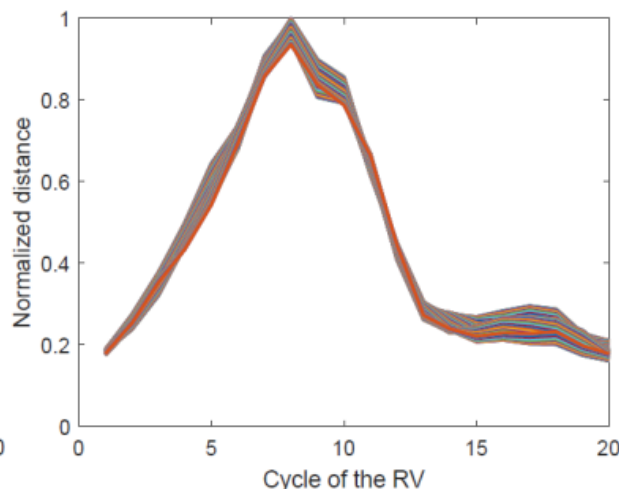

(f)

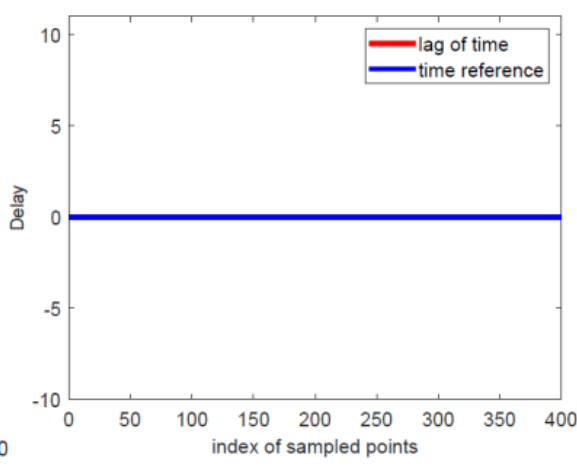

(h)

Fig.11. Comparison of the results of computing the mechanical dyssynchrony for the LV in a typical normal control by the method of [16] and by the proposed approach (a) the computed correspondence trajectories and the identified boundaries for one middle slice by the method of [16]; (b) the cycles of the filtered distances by the method of [16]; (c) the cycle of the 20 areas by the method of [16]; (d) the mechanical dyssynchrony computed from four middle slices by the method of [16]; (e) the computed correspondence trajectories and the identified boundaries for one middle slice by the proposed approach; (f) the cycles of the filtered distances by the proposed approach; (g) the cycle of the 20 areas by the proposed approach; (h) the mechanical dyssynchrony computed from four middle slices by the proposed approach.

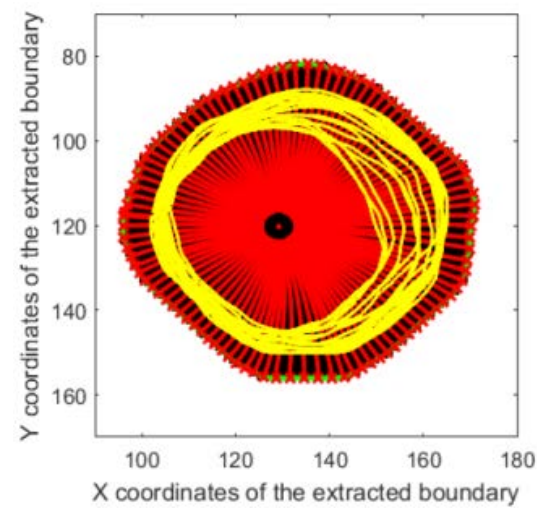

(a)

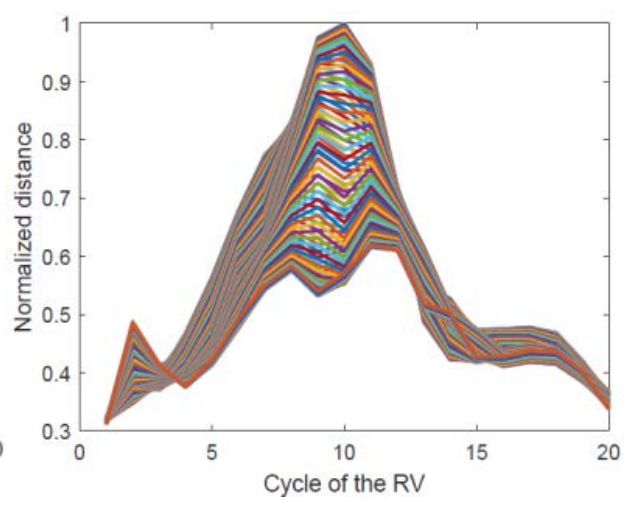

(b) 


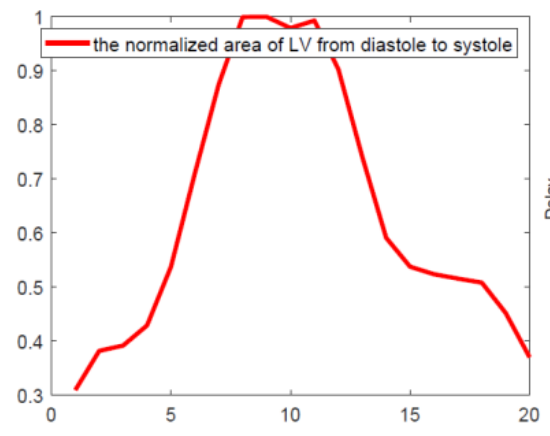

(c)

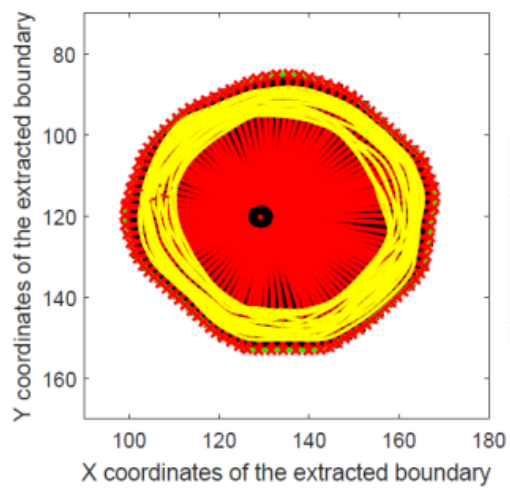

(e)

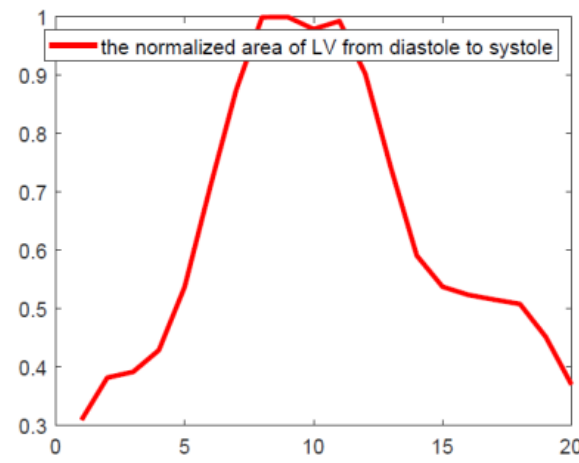

(g)

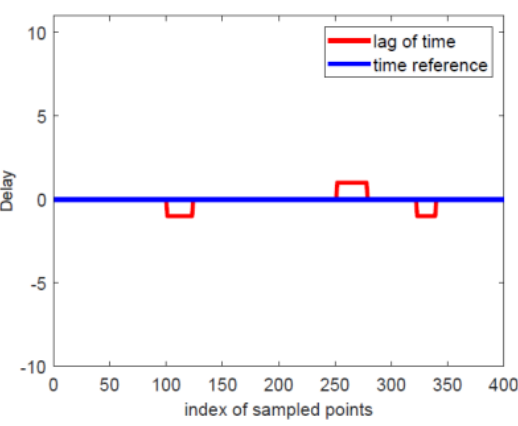

(d)

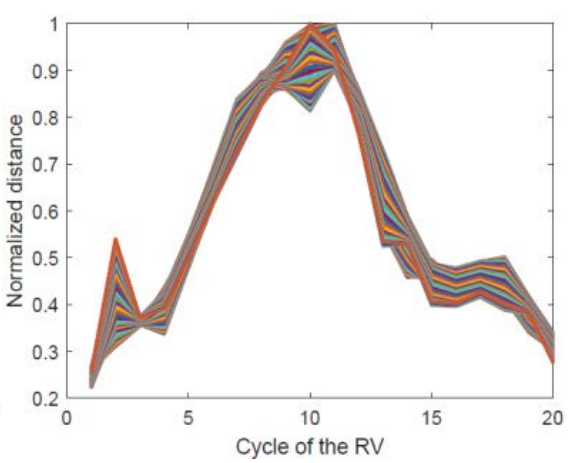

(f)

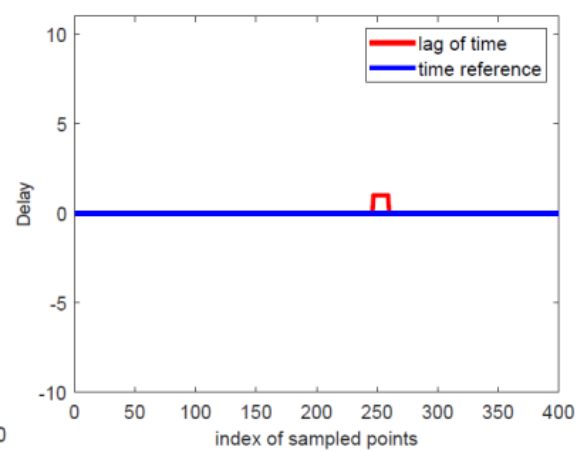

(h)

Fig.12. Comparison of the results of computing the mechanical dyssynchrony for the LV in a typical patient case by the method of [16] and by the proposed approach (a) the computed correspondence trajectories and the identified boundaries for one middle slice by the method of [16]; (b) the cycles of the filtered distances by the method of [16]; (c) the cycle of the 20 areas by the method of [16]; (d) the mechanical dyssynchrony computed from four middle slices by the method of [16]; (e) the computed correspondence trajectories and the identified boundaries for one middle slice by the proposed approach; (f) the cycles of the filtered distances by the proposed approach; (g) the cycle of the 20 areas by the proposed approach; (h) the mechanical dyssynchrony computed from four middle slices by the proposed approach. 


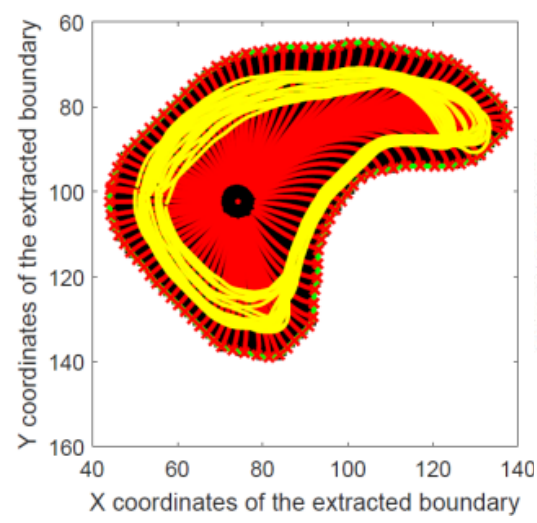

(a)

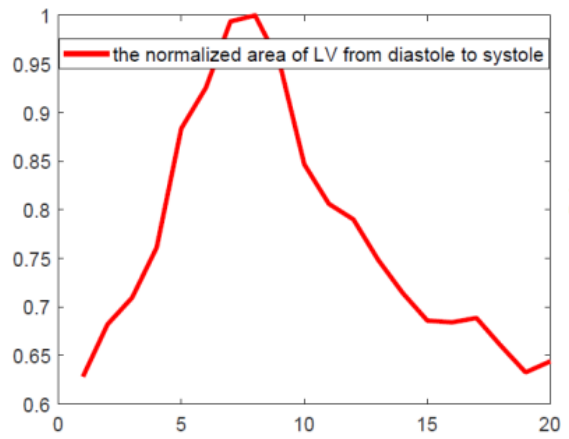

(c)

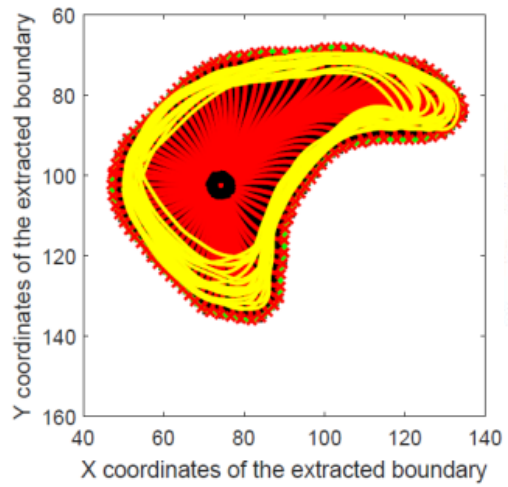

(e)

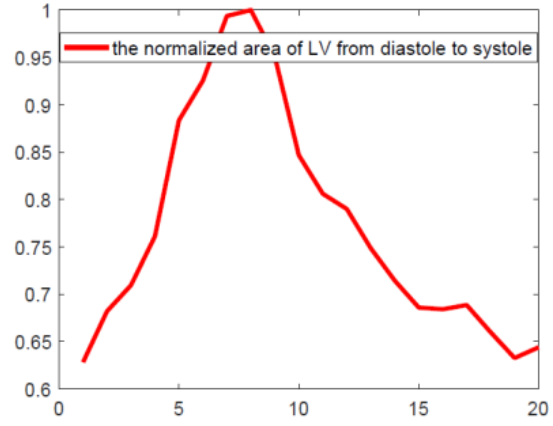

(g)

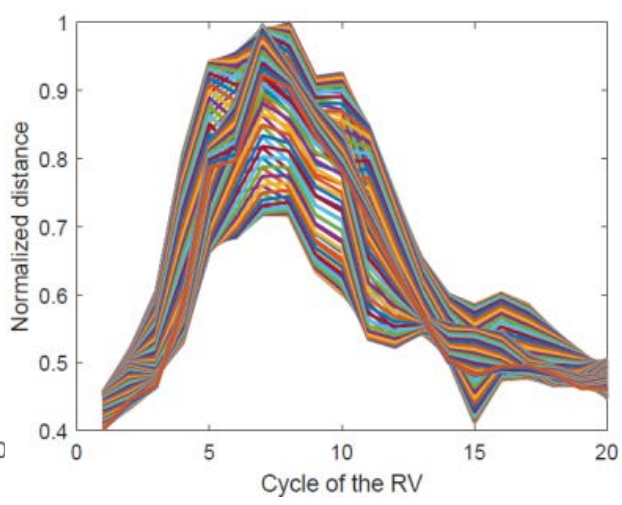

(b)

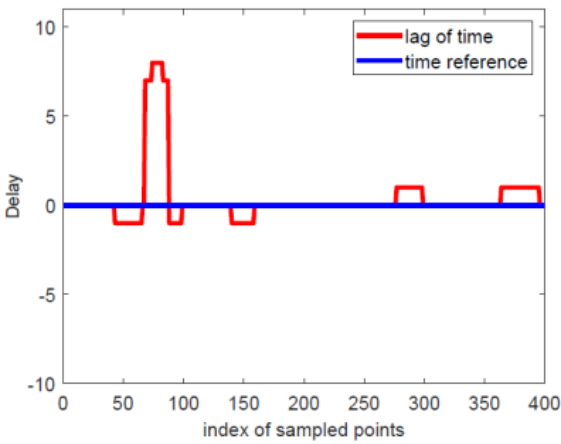

(d)

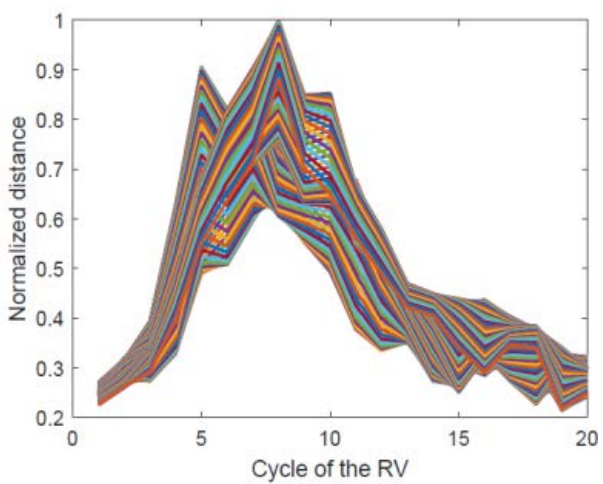

(f)

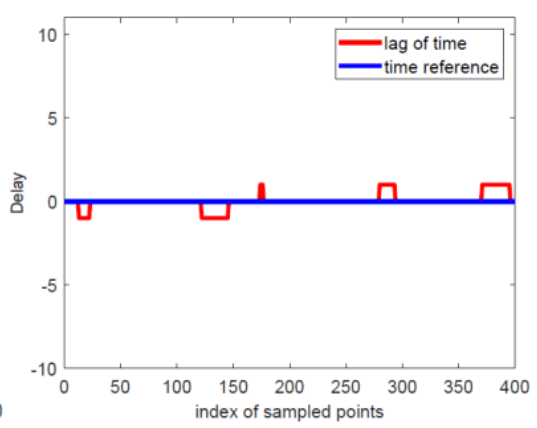

(h) 
Fig.13. Comparison of the results of computing the mechanical dyssynchrony for the RV in a typical normal control by the method of [16] and by the proposed approach (a) the computed correspondence trajectories and the identified boundaries for one middle slice by the method of [16]; (b) the cycles of the filtered distances by the method of [16]; (c) the cycle of the 20 areas by the method of [16]; (d) the mechanical dyssynchrony computed from four middle slices by the method of [16]; (e) the computed correspondence trajectories and the identified boundaries for one middle slice by the proposed approach; (f) the cycles of the filtered distances by the proposed approach; (g) the cycle of the 20 areas by the proposed approach; (h) the mechanical dyssynchrony computed from four middle slices by the proposed approach.

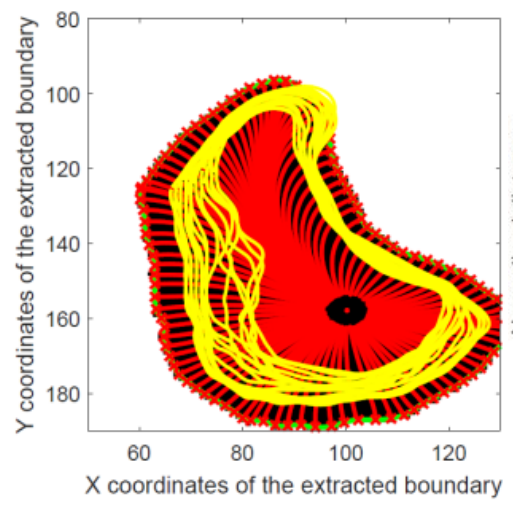

(a)

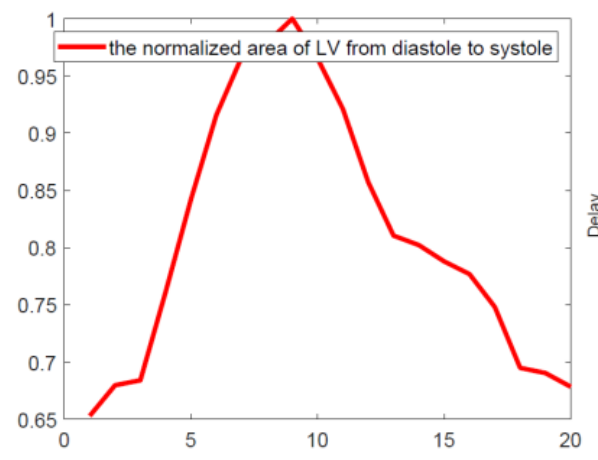

(c)

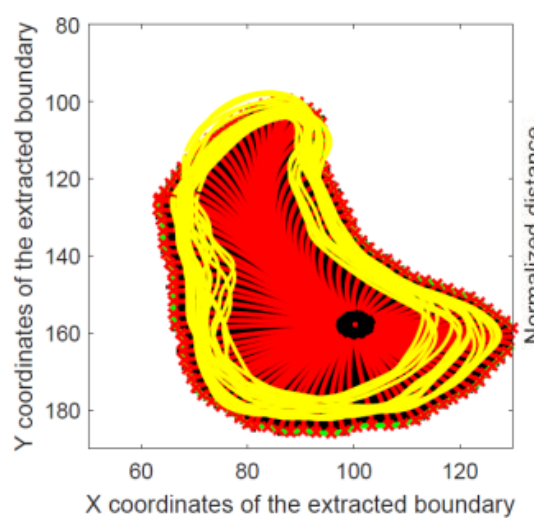

(e)

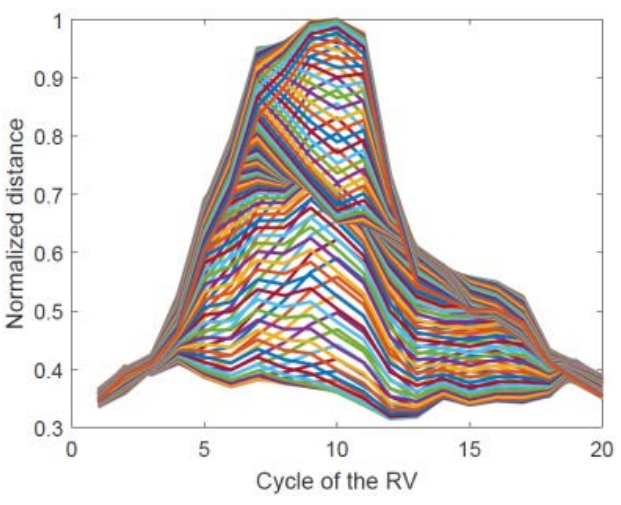

(b)

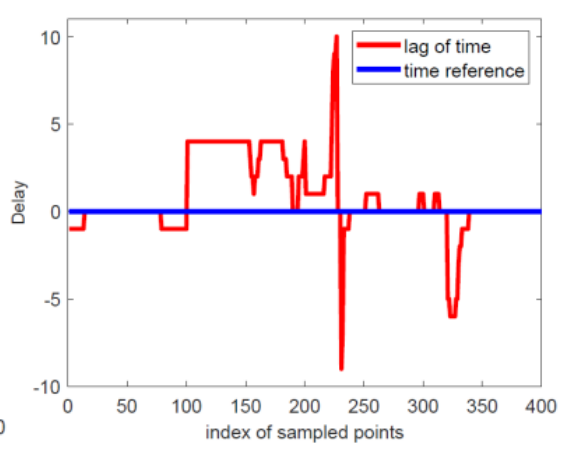

(d)

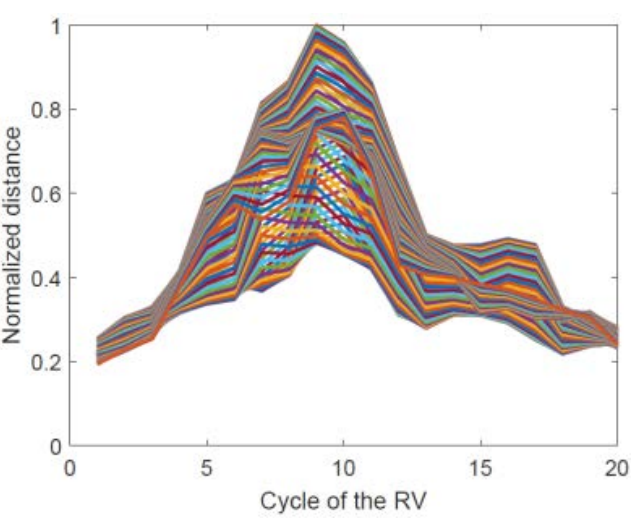

(f) 


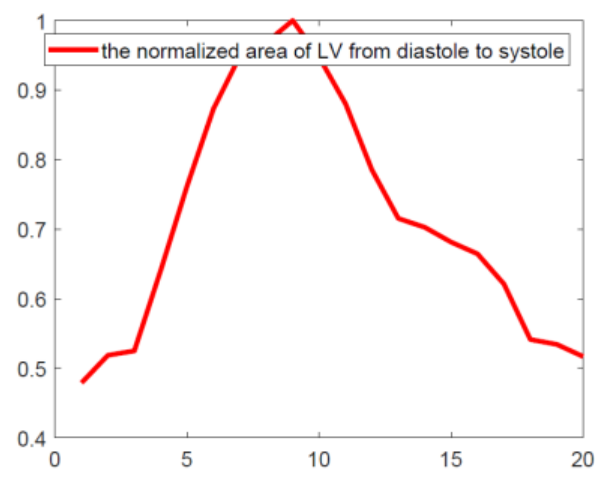

(g)

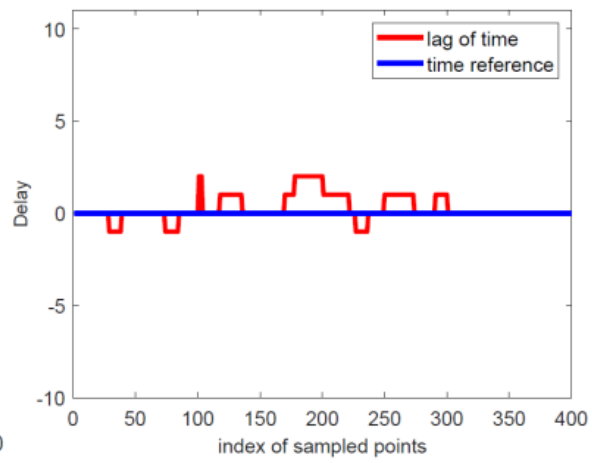

(h)

Fig.14. Comparison of the results of computing the mechanical dyssynchrony for the RV in a typical patient case by the method of [16] and by the proposed approach (a) the computed correspondence trajectories and the identified boundaries for one middle slice by the method of [16]; (b) the cycles of the filtered distances by the method of [16]; (c) the cycle of the 20 areas by the method of [16]; (d) the mechanical dyssynchrony computed from four middle slices by the method of [16]; (e) the computed correspondence trajectories and the identified boundaries for one middle slice by the proposed approach; (f) the cycles of the filtered distances by the proposed approach; (g) the cycle of the 20 areas by the proposed approach; (h) the mechanical dyssynchrony computed from four middle slices by the proposed approach.

\section{Discussion}

Myocardial strain measurement by FT-CMR has become very popular in recent years. However, the measurement results are significantly diverse depending on the technique and the software used [1,7]. It also requires great effort to determine the normal range of the calculated strain for each software before it could be used clinically. Most importantly, the strains are computed based on a limited number of pre-defined segments,which lacks regional information about the motion and defortion of the ventricle. In this paper, a new approach is proposed to measure the mechanical dyssynchrony based on a great number of sampled points. As a result, it could yield both the global and the regional information about the motion and deformation of the ventricle. Based the experimental results of the LV, the normal controls could be distinguished straightforwardly from the patients. No additional efforts are required to determine a normal range for the proposed approach as required by strain based methods [6,7].

Although the proposed approach did not achieve the same 100\% auto-diagnosis accuracy rate in $\mathrm{RV}$ as it did in measuring the mechanical dyssynchrony for the $\mathrm{LV}$, the incorporation of image registration to rectify the effect of heart torsion and displacement will increase the auto-diagnosis rate for the RV significantly.

\section{Conclusion}

In this paper, a fully automatic approach is proposed to measure the mechanical dynssynchrony of both the LV and the RV. The fully automatic SDD segmentation method is used to segment both the LV and the RV robustly. A fully automatic ventricle registration method is proposed to align the boundaries of the ventricle 
robustly. Finally, the intra-ventricular mechanical dyssynchrony of both the LV and the RV are measured robustly and automatically. All the methods are tested with 20 normal controls and 20 patients and experimental results verified the robustness of the all the methods.

\section{Conflicts of Interest}

There is no conflict of interest.

\section{References}

[1] J.J. Cao,N. Ngai, L. Duncanson, J. Cheng, K. Gliganic, Q. Chen. “A comparison of both DENSE and feature tracking techniques with tagging for the cardiovascular magnetic resonance assessment of myocardial strain.” $\mathrm{J}$ Cardiovasc Magn Reson., 20(1):26, 2018.

[2] Scatteia, A. Baritussio, D. C. Bucciarelli. "Strain imaging using cardiac magnetic resonance.” Heart Fail Rev., 22(4):465-476, 2017.

[3] P. Claus, A.M.S. Omar, G. Pedrizzetti, P.P. Sengupta, E. Nagel. “Tissue Tracking Technology for Assessing Cardiac Mechanics: Principles, Normal Values, and Clinical Applications.” JACC Cardiovasc Imaging., 8(12):1444-1460, 2015.

[4] M. Aurich, M. Keller, S. Greiner, H. Steen, S. F. Aus dem, J. Riffel, H. A. Katus, S. J. Buss, D. Mereles. "Left ventricular mechanics assessed by two-dimensional echocardiography and cardiac magnetic resonance imaging: comparison of high-resolution speckle tracking and feature tracking." Eur Heart J Cardiovasc Imaging., 17(12):1370-1378, 2016.

[5] T. Lapinskas, J. Grune, S. M. Zamani, S. Jeuthe, D. Messroghli, R. Gebker, H. Meyborg, U. Kintscher, R. Zaliunas, B. Pieske, P. Stawowy and S. Kelle, "Cardiovascular magnetic resonance feature tracking in small animals - a preliminary study on reproducibility and sample size calculation.” BMC Med Imaging., 17: 51, 2017.

[6] R.J. Taylor, W.E. Moody, F. Umar, N.C. Edwards, T.J. Taylor, B. Stegemann, J.N. Townend, K.N. Hor, R.P. Steeds, W. Mazur, F. Leyva, "Myocardial strain measurement with feature-tracking cardiovascular magnetic resonance: normal values.” Eur Heart J Cardiovasc Imaging., 16(8):871-81, 2015.

[7] K. Takigiku, M. Takeuchi, C. Izumi, S. Yuda, K. Sakata, N. Ohte, K. Tanabe, S. Nakatani, "Normal range of left ventricular 2-dimensional strain: Japanese Ultrasound Speckle Tracking of the Left Ventricle (JUSTICE) study." Circ J. 76(11): 2623-2632, 2012.

[8] A. Schuster, V. C. Stahnke, C. Unterberg-Buchwald, J.T. Kowallick, P. Lamata, M. Steinmetz, S. Kutty, M. Fasshauer, W. Staab, J.M. Sohns, B. Bigalke, C. Ritter, G. Hasenfuß, P. Beerbaum, and J. Lotz, "Cardiovascular magnetic resonance feature-tracking assessment of myocardial mechanics: Intervendor agreement and considerations regarding reproducibility." Clin Radiol., 70(9): 989-998, 2015.

[9] G. Prati, G. Vitrella, G. Allocca, D. Muser, S.C. Buttignoni, G. Piccoli, G. Morocutti, P. Delise,B. Pinamonti, A. Proclemer, G. Sinagra, G. Nucifora. "Right Ventricular Strain and Dyssynchrony Assessment in Arrhythmogenic Right Ventricular Cardiomyopathy: Cardiac Magnetic Resonance Feature-Tracking Study.” Circ Cardiovasc Imaging., 8(11):e003647, 2015 
[10] V. T. Truong, C. Y. Li, R. L. Brown, R. A. Moore, V. F. Garcia, E. J. Crotty, M. D. Taylor, T. M. N. Ngo, W. Mazur, "Occult RV systolic dysfunction detected by CMR derived RV circumferential strain in patients with pectus excavatum.” PLoS One, 12(12): e0189128, 2017.

[11] M. Bourfiss, D.M. Vigneault, G. M. Aliyari, B. Murray, C.A. James, C. Tichnell, H. F. A. Mohamed, S.L. Zimmerman, I. R. Kamel, H. Calkins, H. Tandri, B. K. Velthuis, D. A. Bluemke, R. A. S. J. M. Te, "Feature tracking CMR reveals abnormal strain in preclinical arrhythmogenic right ventricular dysplasia/ cardiomyopathy: a multisoftware feasibility and clinical implementation study.” J Cardiovasc Magn Reson., 19(1):66, 2017.

[12] S. F. nagueh, "Mechanical dyssynchrony in congestive heart failure: diagnostic and therapeutic implications.” J Am Coll Cardiol., 51(1):18-22, 2008.

[13] B.K. Fornwalt, W.W. Sprague, P. Bedell, J.D. Suever, B. Gerritse, J.D. Merlino, D.A. Fyfe, A.R. Leon and J.N. Oshinski,"Agreement is poor among current criteria used to define response to cardiac resynchronization therapy," Circulation. Vol. 121, pp.1985-1991, 2010

[14] B.K. Fornwalt, J.G. Delfino, W.W. Sprague and J.N. Oshinski,"It's time for a pradigm shift in the quantitative evaluation of left ventricular dyssynchrony," JASE. pp.672-676, 2009

[15] N. M. Hawkins, M.C. Petrie, M.R. Macdonald, K.J. Hogg and H. J. V. Mcmurray,"Selecting patients for cardiac resynchronization therapy: electrical or mechanical dyssynchrony," European Heart Journal. Vol. 27, pp.1270-1281, 2006

[16] Z.Z. Wang, " Robust and automatic diagnosis of the intraventricular mechanical dyssynchrony for the left ventricle in cardiac magnetic resonance images," Int J CARS., 12(9):1471-1480,2017.

[17] Z.Z. Wang, "Automatic and Optimal Segmentation of the Left Ventricle in Cardiac Magnetic Resonance Images Independent of the Training Sets,” IET image processing, Vol. 13, Issue, 10, pp. 1725-1735, 2019.

[18] Z.Z. Wang, "An efficient and robust method for automatically identifying the left ventricular boundary in cine magnetic resonance images," IEEE Trans. ASE. Vol. 13, No. 2, pp. 536-542, 2016

[19] Ming L, Shizhou D, Zhifan G, Cheng F, et al .Unified model for interpreting multi-view echocardiographic sequences without temporal information. Applied Soft Computing Journal, 88:106049, 2020.

[20] Zhifan G, Geye Z, Shizhou Dong, Shanhui S, et al. Salient Object Detection in the Distributed Cloud-Edge Intelligent Network. IEEE Network, 1-9, 2020

[21] Dingwen Z, Deyu M, Chao L, Jiang L. Co-saliency detection via a self-paced multiple-instance learning framework. 2015 IEEE International Conference on Computer Vision, 2015.

[22] Dingwen Z, Junwei H, Chao L, et al. Detection of Co-salient Objects by Looking Deep and Wide. International journal of computer vision, 120:215-232, 2016.

[23] Marcus A.G. Santos, Roberto Munoz, Rodrigo Olivares, et al. Online heart monitoring systems on the internet of health things environments: A survey, a reference model and an outlook. Information Fusion, 53:222-239, 2020.

[24] Victor Hugo C. de Albuquerque, Douglas de A. Rodrigues, et al. Fast fully automatic heart fat segmentation in computed tomography datasets. Computerized Medical Imaging and Graphics, 80:101674, 2020. 
[25] Leandro B. Marinho, Navar de M.M. Nascimento, João Wellington M. Souza, et al. A novel electrocardiogram feature extraction approach for cardiac arrhythmia classification. Future Generation Computer Systems, 91:564-577, 2019.

[26] Ahmed Faeq Hussein, ArunKumar N, Gustavo Ramirez-Gonzalez, et al. A Medical Records Managing and Securing Blockchain Based System Supported by a Genetic Algorithm and Discrete Wavelet Transform. Cognitive Systems Research, 52:1-11, 2018.

[27] Jermana L. Moraes, Matheus X. Rocha, Glauber G. Vasconcelos, et al. Advances in Photoplethysmography Signal Analysis for Biomedical Applications. Sensors, 18(6):1894, 2018.

[28] Jude D. Hemanth, Utku Kose, Omer Deperlioglu, Victor Hugo C and de Albuquerque. An augmented reality-supported mobile application for diagnosis of heart diseases. The Journal of Supercomputing, 76:1242-1267, 2020

[29] Ahmed Faeq Hussein, Arun Kumar N, Marlon Burbano-Fernandez, Gustavo Ramirez-Gonzalez, et al. An Automated Remote Cloud-Based Heart Rate Variability Monitoring System. IEEE Access, 1:99, 2018.

[30] Auzuir Ripardo, De Alexandria, A. R. Cortez, P. C. Bessa, et al. pSnakes:a new radial active contour model and its application in the segmentation of the left ventricle from echocardiographic images. Computer Methods and Programs in Biomedicine,

[31] Yankun C, Zhi L, Pengfei Z, Yushuo Z, et al. Deep Learning Methods for Cardiovascular Image. Journal of Artificial Intelligence and Systems, 1:96-109, 2019.

[32] Aldisio G. Medeiros, Francisco H. S. Silva, Elene F. Ohata, et al. An Automatic Left Ventricle Segmentation on Echocardiogram Exams via Morphological Geodesic Active Contour with Adaptive External Energy. Journal of Artificial Intelligence and Systems, 1: 77-95, 2019. 